\title{
Quilotórax associado à torção de lobo pulmonar em gata
}

\author{
Chylothorax associated with lung lobe torsion in a cat \\ Raquel Calixto ${ }^{1}$, Heloisa J usten Moreira de Souzaa $\&$ Katia Barão Corgozinho ${ }^{1}$
}

\begin{abstract}
RESUMO
A torção de lobo pulmonar (TLP) é uma enfermidade rara em cães e excepcional em gatos. Os lobos mais afetados são os médios direito e esquerdo, embora qualquer lobo possa sofrer uma torção, sendo que sua etiologia é pouco compreendida. O quilotórax é o acúmulo de fluido linfático na cavidade torácica, que ocorre com grande frequiência em gatos, e está freqüentemente associado à TLP, mas ainda não foi esclarecido se ele é a causa ou a consequiência da torção. Os sinais clínicos incluem tosse, prostração, taquipnéia, dispnéia, anorexia, vômito, e diarréia. Uma gata, sem raça definida, esterilizada com 13 anos de idade foi encaminhada para clínica veterinária particular (Gatos \& Gatos Vet.) no Rio de Janeiro, apresentando intensa dispnéia, anorexia, prostração. O estudo radiográfico evidenciou efusão pleural grave, e aumento da radiopacidade cranial após drenagem do fluido. Procedeu-se a colocação de dreno torácico e após a estabilização do animal, foi realizada toracotomia, que revelou a TLP médio esquerdo, sendo feita à lobectomia. do mesmo. Cerca de três semanas após o primeiro atendimento foi retirado o dreno, mas o animal não resistiu vindo a óbito após quatro semanas.
\end{abstract}

Descritores: torção de lobo pulmonar, quilotórax, gato.

\section{ABSTRACT}

Lung Lobe Torsion is an extremely rare condition in dogs and cats. The most commonly affected lobes are right and left middle lung lobes, although any lobe can be affected. The etiology of lung lobe torsion is still unclear. Chylothorax is the accumulation of chylous fluid in pleural cavity. Chylothorax occurs frequently in cats, and it is commonly associated with lung lobe torsion. However, it has not been clarified if it is the cause or a consequence of torsion. Clinical signs include coughing, weakness, tachypnea, dyspnea, anorexia, vomit and diarrhea. A 13-year-old spayed domestic shorthair female cat was referred to private veterinary clinic (Gatos \& Gatos Vet.) in Rio de Janeiro, for dyspnea, anorexia and weakness. Pleural effusion was present and post-drainage radiographs showed cranial lung lobe density. After chest drain placement and patient stabilization, an exploratory thoracotomy was performed, which revealed left middle lung lobe torsion. A lobectomy was then performed. Three weeks later, the chest drain was removed, but the cat died after four weeks.

Key words: lung lobe torsion, chylothorax, cat. 


\section{INTRODUÇãO}

A torção de lobo pulmonar (TLP) é uma enfermidade excepcional em gatos com apenas sete casos relatados [5]. Os lobos mais afetados são os médios direito e esquerdo [5,7]. A torção acontece quando o lobo pulmonar rotaciona em torno do pedículo broncovascular de forma irreversível. As veias facilmente se obliteram, e as artérias mantêm seu fluxo para o interior do pulmão, resultando em severa congestão e consolidação, associadas à efusão pleural.

A etiologia da TLP é pouco compreendida. Acredita-se na associação que seja provocada por efusão pleural, pneumotórax, trauma, pneumonia ou manipulação cirúrgica, com aumento de ar ou fluido ao redor do lobo, predispondo à sua rotação. A TLP espontânea já foi diagnosticada em cães e gatos sem a identificação de nenhum fator predisponente. Os sinais clínicos incluem tosse, prostração, taquipnéia, dispnéia, anorexia, vômito, e diarréia.

O diagnóstico é realizado através de radiografias torácicas, onde se pode visualizar um ou mais lobos pulmonares consolidados. A ultra-sonografia de tórax tem sido mais utilizada para o diagnóstico da TLP [5]. As alterações histopatológicas incluem desde acúmulo de fluido hemorrágico no brônquio, trombose venosa, infiltração de plasmócitos e linfócitos até necrose.

O tratamento da TLP é feito através da remoção cirúrgica do lobo afetado. É realizada toracotomia lateral. O pedículo broncovascular é cuidadosamente ligado para evitar a liberação de citoquinas na circulação sistêmica, e remove-se o lobo afetado. A efusão pleural deve ser drenada diariamente até que haja menos de $5 \mathrm{ml} / \mathrm{Kg} /$ dia de fluido sendo produzido [5]. O objetivo desse trabalho é relatar um caso extremamente raro de quilotórax associado à TLP.

\section{RELATO DE CASO}

Uma gata, sem raça definida, esterilizada, com 13 anos de idade (Figura 1) foi encaminhada à Clínica Veterinária Gatos \& Gatos Vet., Rio de Janeiro, apresentando intensa dispnéia, anorexia, prostração e efusão pleural grave há aproximadamente 21 dias. O proprietário apresentou os seguintes exames anteriormente realizados: ecocardiograma, o qual constatou cardiomiopatia hipertrófica com efusão pleural leve; e análise da efusão pleural, revelando um exsudato séptico; hemograma, pesquisa de hemoparasitos, bioquímica hepática e renal, e ainda sorologia para coronavírus e teste ELISA para detecção do antígeno para o vírus da Leucemia Felina (FeLV) e do anticorpo do vírus da Imunodeficiência Felina (FIV). Esses dois últimos se revelaram negativos, bem como a pesquisa para hemoparasitos. A sorologia para coronavírus mostrouse positiva com baixa titulação. O hemograma demonstrou uma leucopenia (4.400 leucócitos) com linfopenia (308 linfócitos). Os exames bioquímicos não revelaram alterações.

Ao exame clínico, o felino apresentava dispnéia com padrão respiratório abdominal e taquipnéia com freqüência respiratória de 80 movimentos respiratórios por minuto (mrm). A ausculta pulmonar revelou silêncio auscultatório em ambos hemitórax e a ausculta cardíaca revelou ruídos reduzidos. A frequiência cardíaca encontrava-se em 186 batimentos por minuto (bpm). A gata estava em bom estado corpóreo $(3,35 \mathrm{Kg})$ e com grau de desidratação de $7 \%$, porém com temperatura corpórea baixa $\left(36,4^{\circ} \mathrm{C}\right)$.

Radiografias de tórax nas incidências láterolateral e ventro-dorsal em posição ortostática foram feitas e constatou-se efusão pleural grave em ambos hemitórax. Realizou-se a toracocentese, e colocação de dreno torácico, onde o felino foi anestesiado. Foram drenados $112 \mathrm{ml}$ do hemitórax esquerdo e $60 \mathrm{ml}$ do hemitórax esquerdo de líquido levemente róseo e turvo, cujas análises citológica e bioquímica revelaram efusão quilosa. Após a remoção da efusão, o exame radiográfico, nas mesmas posições e incidências anteriormente citadas, demonstrou extenso aumento da radiopacidade na região cranial do hemitórax esquerdo (Figura 2).

Realizado também hemograma, dosagem de proteínas totais séricas e frações, dosagem de colesterol e triglicerídeos séricos, bem como do líquido cavitários e ultra-sonografia do tórax. Os resultados mostraram uma neutrofilia (17.480 neutrófilos segmentados) e linfopenia (368 linfócitos) absolutas e ainda, hipoalbuminemia $(1,7 \mathrm{mg} / \mathrm{dl})$. As concentrações de colesterol e triglicerídeos séricos $(129,6 \mathrm{mg} / \mathrm{dl}$ e 52,9 $\mathrm{mg} / \mathrm{dl}$, respectivamente) estavam inferiores às mesmas concentrações do líquido cavitário $(58,9 \mathrm{mg} / \mathrm{dl}$ e 313,4 $\mathrm{mg} / \mathrm{dl}$, respectivamente). $\mathrm{O}$ exame ultra-sonográfico revelou massa hipoecóica no parênquima pulmonar esquerdo, medindo cerca de $5,0 \times 1,5 \mathrm{~cm}$, junto ao coração sugestivo de neoplasia. 
Adotou-se como tratamento de suporte: ampicilina $(20 \mathrm{mg} / \mathrm{Kg}$, por via oral, a cada 8 horas), meloxicam $(0,1 \mathrm{mg} / \mathrm{Kg}$, por via oral, a cada 24 horas, durante 5 dias), tramadol $(2 \mathrm{mg} / \mathrm{Kg}$, por via oral, a cada 12 horas, durante 5 dias $)$ e rutina $(50 \mathrm{mg} / \mathrm{Kg}$, por via oral, a cada 12 horas). Além disso, uma terapia nutricional involuntária por via oral foi instituída, utilizando-se ração de baixo teor de gordura.

Realizada citologia da massa tumoral por punção aspirativa por agulha fina, cujo resultado revelou amostra era de baixa celularidade, onde foi encontrado apenas material basofílico com células "fantasmas", com raros macrófagos altamente vacuolizados e neutrófilos, além de resquícios de células escamosas descamativas e queratina, compatível com necrose.

Após sete dias, o felino foi submetido à toracotomia exploratória, utilizando-se o seguinte protocolo anestesiológico: diazepam na dosagem de $0,2 \mathrm{mg} / \mathrm{Kg}$ e meperidina na dosagem de $2,0 \mathrm{mg} / \mathrm{Kg}$ ambos por via intramuscular (IM) como medicação pré-anestésica; etomidato na dosagem de $2,0 \mathrm{mg} / \mathrm{Kg}$ por via endovenosa (EV) como medicação para indução e manutenção com isoflurano. Administrou-se ainda atropina na dosagem de $0,04 \mathrm{mg} / \mathrm{Kg}$ por via $\mathrm{EV}$ e meloxicam na dosagem de $0,2 \mathrm{mg} / \mathrm{Kg}$ por via subcutânea (SC). Realizou-se a toracotomia lateral esquerda, onde se visualizou o lobo médio com coloração acinzentada, consolidado e não inflável (Figura 3), procedeu-se a lobectomia.

Coletado material para realização de exame histopatológico, o qual revelou pleurite associada à necrose pulmonar difusa, hemorragia, infarto pulmonar e pneumonia (Figuras 4 e 5). Sete dias após a cirurgia, o animal apresentava episódios de vômito, continuava prostrado e inapetente. Continuava-se a drenar fluido pleural com o mesmo aspecto inicial, porém em menor quantidade, aproximadamente $30 \mathrm{ml}$ ao dia. Após dois dias, a quantidade de líquido drenada era ainda menor, cerca de $6 \mathrm{ml}$ ao dia, mediante a isso, optou-se pela retirada do dreno torácico. Todavia, o felino demonstrava emagrecimento progressivo, mucosas hipocoradas e desidratação persistente, e em função da confluência de fatores debilitantes, como severo déficit nutricional causado pelo quilotórax, pneumonia preexistente, e o procedimento cirúrgico, o animal veio a óbito quatro semanas após o primeiro atendimento.

\section{DISCUSSÃO}

A torção de lobo pulmonar (TLP) é uma condição muito rara em gatos [3,5], e afeta mais freqüentemente os lobos médios direito ou esquerdo, havendo efusão pleural. Tal patologia está associada a doenças preexistentes como quilotórax, piotórax, linfoma mediastinal, cardiopatias, bronquite e asma [2,3,7]. Pneumonia foi a doença pulmonar preexistente encontrada neste caso, todavia, ressalta-se a importância do quilotórax e da cardiomiopatia hipertrófica como fatores predisponentes para a TLP nesse caso. Existem somente sete casos de TLP na literatura, sendo apenas dois deles associados ao quilotórax [2-4]. Os sinais clínicos da TLP são inespecíficos e incluem: tosse, dispnéia, taquipnéia, prostração, inapetência [5,7], como neste relato. E o tipo de efusão pleural é variável, dependendo da enfermidade preexistente [5,7]. O diagnóstico presuntivo é efetuado através de radiografias, ultra-sonografia e broncoscopia [2,6,7], mas sua confirmação é feita através da histopatologia [2]. Neste caso, a suspeita clínica foi baseada em possível neoplasia através das alterações radiográficas e ultra-sonográficas, entrentanto, a possibilidade de TLP foi levantada a partir das alterações visualizadas no transoperatório, e firmada, posteriomente, pelo exame histopatológico. $\mathrm{O}$ tratamento envolve a estabilização do paciente com oxigenioterapia e drenagem do fluido pleural para o alívio da dispnéia, e também reposição hidro-eletrolítica. Entretanto, a excisão cirúrgica do lobo afetado é necessária para o tratamento definitivo [5]. A terapia para o quilotórax é baseada na correção da causa, na terapia nutricional com redução da ingestão de gorduras, e a permanência de dreno torácico para retirada diária do fluido [4,5]. Tais procedimentos foram realizados neste caso para estabilização do paciente até a remoção do lobo afetado. O diagnóstico é baseado na eliminação de outras patologias mais frequientes do trato respiratório inferior, e confirmado somente pela histopatologia. Dos dois casos relatados na literatura de quilotórax associado à TLP, somente um animal sobreviveu, obtendo boa qualidade de vida até um ano após a cirurgia [4]; no outro caso o animal foi submetido à eutanásia sem que um tratamento pudesse ser igualmente instituído [2]. Neste caso, o animal não sobreviveu às complicações da enfermidade após aproximadamente 30 dias do primeiro atendimento. 


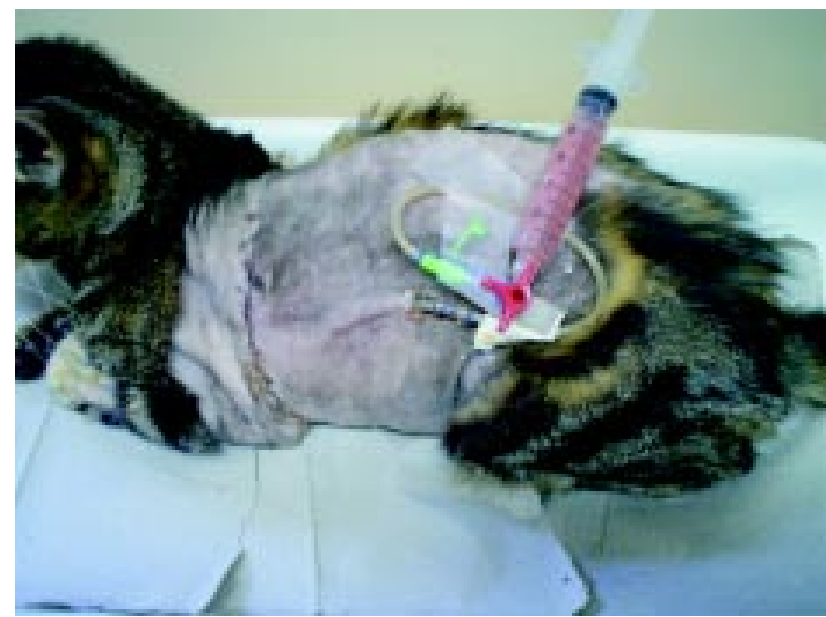

Figura 1. Gata com dreno torácico.

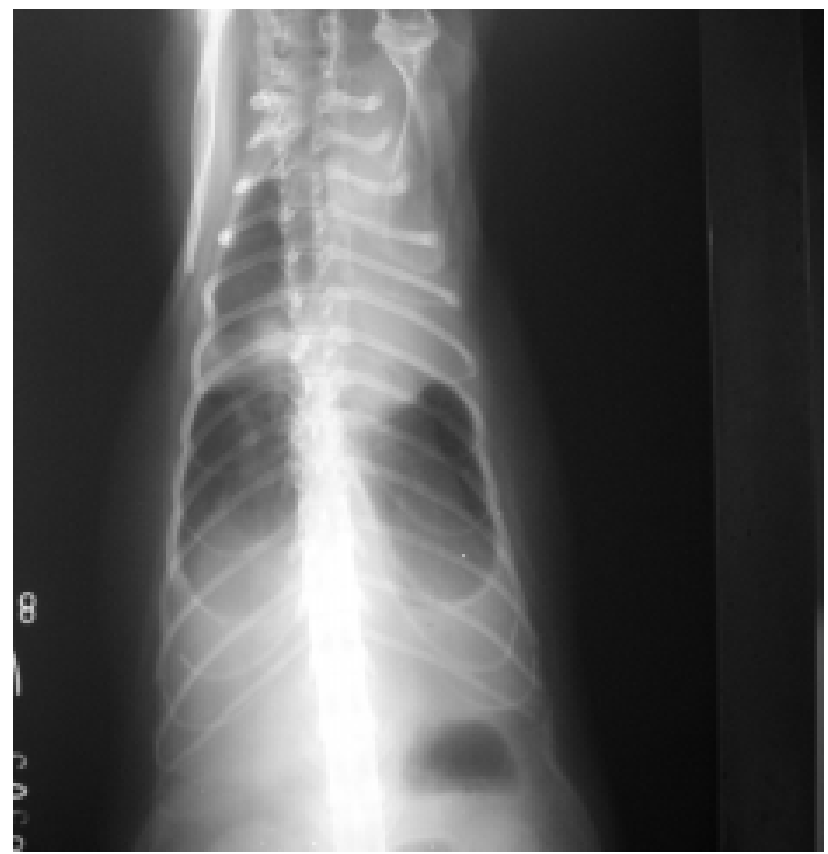

Figura 2. Radiografia de tórax, incidência ventro-dorsal. Notar aumento da radiopacidade na região cranial.

\section{CONCLUSÃO}

Este é o oitavo caso de torção de lobo pulmonar em gato relatado na literatura, patologia extremamente rara, principalmente quando associada ao quilotórax. São inúmeras as causas de efusões pleurais, dispnéia e taquipnéia em felinos, somente a drenagem do fluido e exames complementares, como radiografias, análise do líquido pleural e histopatologia podem esclarecer qual enfermidade é causadora desse grave quadro respiratório. Não obstante, atenção especial deve ser dada à manutenção da hematose, dando adequado suporte ao animal enfermo.

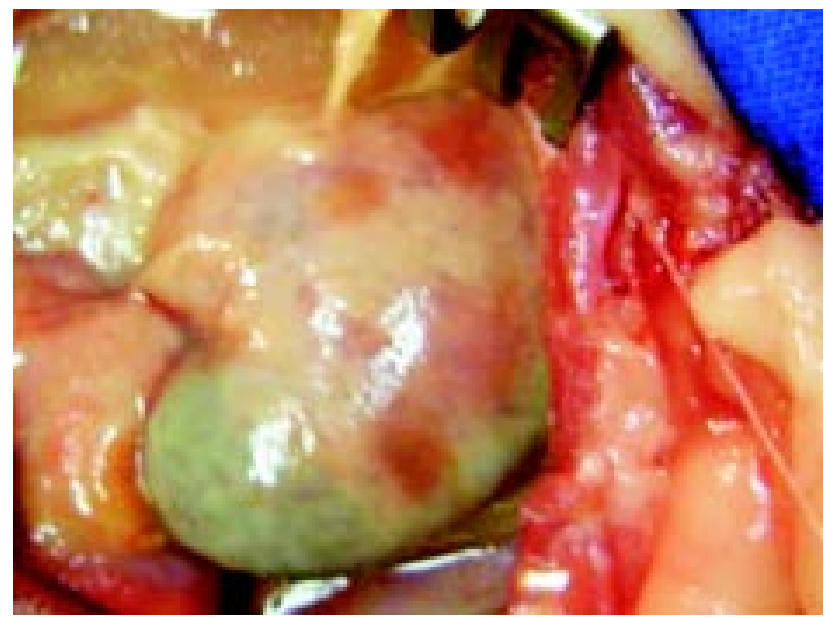

Figura 3. Aspecto macroscópico do lobo pulmonar torcido.

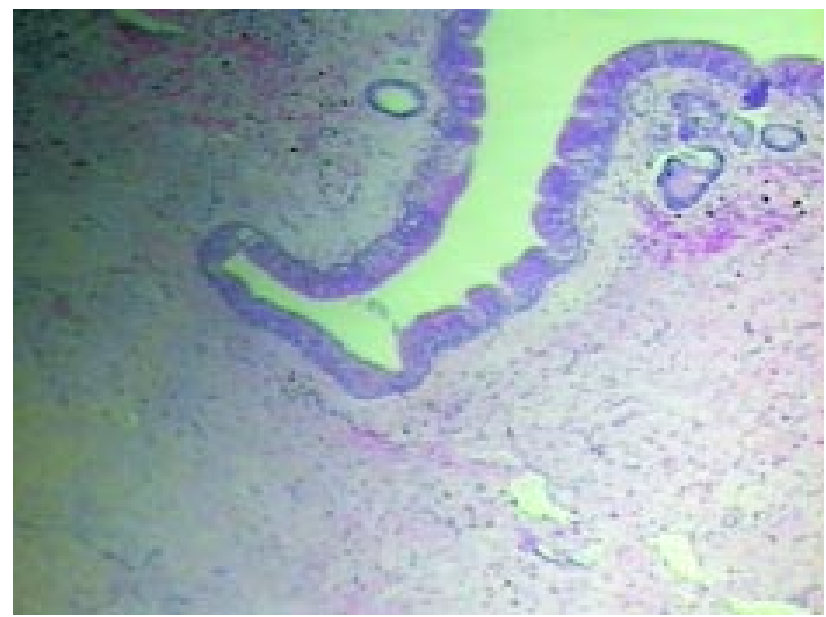

Figura 4. Bronquíolo comáreas de infarto. Hematoxilina-eosina. (400x).

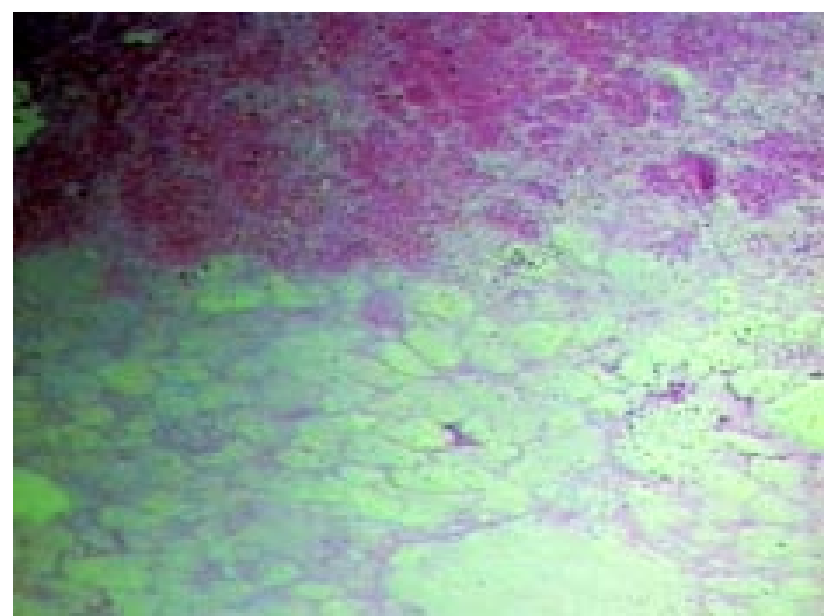

Figura 5. Área de hemorragia pulmonar. Hematoxilina-eosina. (400x). 


\section{REFERÊNCIAS}

1 Fossum T.W. 2001. Chylothorax. In: August J.R. (Ed). Consultations in Feline Internal Medicine. Philadelphia: WB Saunders, pp.267-276.

2 Brown N.O. \& Zontine W.J. 1976. Lung lobe torsion in the cat. American Veterinary Radiology Society. 17: $219-223$.

3 Dye T.L., Teague H.D. \& Poundstone M.L. 1998. Lung lobe torsion in a cat with chronic feline asthma. Journal of the American Animal Hospital Association. 34: 493-495.

4 Kerpsack S.J., McLoughlin M.A., Greves T. K., Smeak D.D., Biller D. \& Leake L. 1994. Chylothorax associated with lung lobe torsion and a peritoneopericardial diaphragmatic hernia in a cat. Journal of the American Animal Hospital Association. 30: 351-354.

5 Neath P.J. 2004. Lung lobe torsion. In: King L.G. (Ed). Textbook of Respiratory Disease in Dogs and Cats. Michigan: Saunders, pp.559-569.

6 Reichle J.K. \& Wisner E.R. 2000. Non-cardiac thoracic ultrasound in 75 feline and canine patients. Veterinary Radiology Ultrasound. 41: 154-162.

7 Sherding R.G. 1998. Disease of the pleural cavity. In: Sherding R.G. (Ed). The Cat Disease and Clinical Management. Philadelphia: W.B. Saunders, pp.1053-1091. 